\title{
Ultrasonic Perfluorohexane-Loaded Monocyte Imaging Toward a Minimally Invasive Technique for Selective Detection of Liver Inflammation in Fatty Liver Disease
}

Citation for published version (APA):

Reesink, K. D., Hendrikx, T., van Gorp, P. J., Hoeks, A. P., \& Shiri-Sverdlov, R. (2018). Ultrasonic Perfluorohexane-Loaded Monocyte Imaging Toward a Minimally Invasive Technique for Selective Detection of Liver Inflammation in Fatty Liver Disease. Journal of Ultrasound in Medicine, 37(4), 921-933. https://doi.org/10.1002/jum.14432

Document status and date:

Published: 01/04/2018

DOI:

10.1002/jum.14432

Document Version:

Publisher's PDF, also known as Version of record

Document license:

Taverne

Please check the document version of this publication:

- A submitted manuscript is the version of the article upon submission and before peer-review. There can be important differences between the submitted version and the official published version of record.

People interested in the research are advised to contact the author for the final version of the publication, or visit the DOI to the publisher's website.

- The final author version and the galley proof are versions of the publication after peer review.

- The final published version features the final layout of the paper including the volume, issue and page numbers.

Link to publication

\footnotetext{
General rights rights.

- You may freely distribute the URL identifying the publication in the public portal. please follow below link for the End User Agreement:

www.umlib.nl/taverne-license

Take down policy

If you believe that this document breaches copyright please contact us at:

repository@maastrichtuniversity.nl

providing details and we will investigate your claim.
}

Copyright and moral rights for the publications made accessible in the public portal are retained by the authors and/or other copyright owners and it is a condition of accessing publications that users recognise and abide by the legal requirements associated with these

- Users may download and print one copy of any publication from the public portal for the purpose of private study or research.

- You may not further distribute the material or use it for any profit-making activity or commercial gain

If the publication is distributed under the terms of Article 25fa of the Dutch Copyright Act, indicated by the "Taverne" license above, 


\title{
Ultrasonic Perfluorohexane-Loaded Monocyte Imaging
}

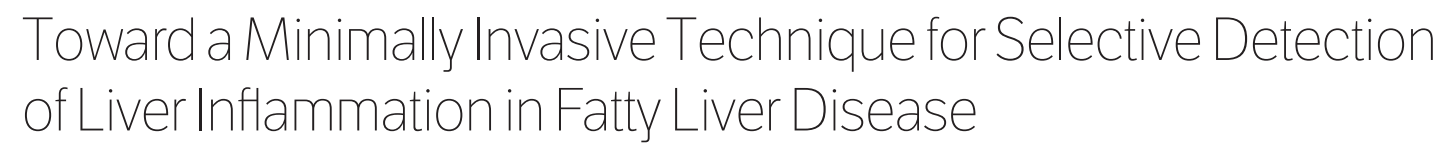

\author{
Koen D. Reesink, PhD, Tim Hendrikx, PhD (D), Patrick J. van Gorp, BASc, Arnold P. Hoeks, PhD, \\ Ronit Shiri-Sverdlov, PhD
}

Supplemental material online at jultrasoundmed.org

Received November 23, 2016, from the Department of Biomedical Engineering, Cardiovascular Research Institute Maastricht School for Cardiovascular Diseases (K.D.R., A.P.H.), and Department of Molecular Genetics, Nutrition and Toxicology Research Institute Maastricht School for Nutritional Toxicology and Metabolism (T.H., P.J.v.G., R.S.-S.), Maastricht University, Maastricht, the Netherlands. Manuscript accepted for publication July 13, 2017.

We thank Agnieszka Brouns-Strzelecka, Jacques Debets, and Peter Leenders from the Department of Pharmacology and Toxicology for skillful animal surgery, care, and handling, and Samantha Scheepers and Lennart Huizing for contributions to the development of experimental and image analysis protocols and support of preliminary experiments. This research was supported by grant WO10-65 from the Dutch Maag-Lever-Darm Stichting (http:// www.mlds.nl/) to Drs Reesink and ShiriSverdlov. The funders had no role in the study design, data collection and analysis, decision to publish, or preparation of the manuscript.

Address correspondence to Koen D. Reesink, PhD, Department of Biomedical Engineering, Maastricht University, PO Box 616, 6200 MD Maastricht, the Netherlands.

E-mail:k.reesink@maastrichtuniversity.nl

Abbreviations

ECG, electrocardiographic; Icam, intracellular adhesion molecule; Itgam, integrin $\alpha M$; NASH, nonalcoholic steatohepatitis; NIMP, antineutrophil antibody; $P F H$, perfluorohexane; PBS, phosphate-buffered saline; ROI, region of interest; TNF, tumor necrosis factor; US, ultrasonic; Vcam, vascular cell adhesion molecule

doi: $10.1002 /$ jum. 14432
Objectives - To investigate the utility of ultrasonic (US) perfluorohexane (PFH)loaded monocyte imaging for detection of liver inflammation in fatty liver disease.

Methods-C57Bl6 mice were injected intraperitoneally with tumor necrosis factor $\alpha$ and assessed by US PFH-loaded monocyte imaging 3 hours later. Echogenic monocytes were injected intravenously, leading to a transient increase in liver tissue intensity on a US perfusion scan. The contrast wash-out time constant was hypothesized to reflect the degree of inflammation. Next, we evaluated US PFH-loaded monocyte imaging in $\mathrm{Ldlr}^{-/-}$mice fed a 1-week high-fat/high-cholesterol diet as model for early developing nonalcoholic steatohepatitis. Adjunct analyses included tissue markers of liver inflammation.

Results - Tumor necrosis factor $\alpha$-injected mice showed a reduced wash-out time constant (mean \pm SEM, $0.013 \pm 0.003 ; \mathrm{n}=8$ ) compared to controls $(0.054 \pm 0.009 ; \mathrm{n}=7 ; P=.0006)$, indicative of increased inflammatory adhesion molecule expression on the endothelium. The $\mathrm{Ldlr}^{-/}$mice fed the highfat/high-cholesterol diet showed liver inflammation, as reflected by increased (3to 4-fold) infiltration of inflammatory cells and increased (3- to 4-fold) gene expression of tumor necrosis factor $\alpha$, integrin $\alpha \mathrm{M}$, intracellular adhesion molecule, and vascular cell adhesion molecule. However, in these mice, no difference was detected in the wash-out time constant as assessed by US PFH-loaded monocyte imaging (high-fat/high-cholesterol, $0.050 \pm 0.017 ; \mathrm{n}=5$; chow, $0.048 \pm 0.006 ; \mathrm{n}=6 ; P=.91)$.

Conclusions - Our results indicate that US PFH-loaded monocyte imaging is able to detect vascularly expressed inflammatory adhesion molecules in the mouse liver on direct endothelial stimulation. However, in our mouse model of early developing nonalcoholic steatohepatitis, we did not detect inflammation by this method, which may suggest that the time-dependent relationship between parenchymal and endothelial inflammation remains a fundamental issue to be addressed.

Key Words - endothelium; animal studies; basic science; fatty liver; gastrointestinal; inflammation; macrophage; monocyte; nonalcoholic steatohepatitis; noninvasive; perfusion; therapeutic; ultrasound contrast

$\mathrm{N}$ onalcoholic fatty liver disease is a chronic liver disorder ranging from benign hepatic lipid accumulation (steatosis) to steatosis combined with inflammation, the latter termed nonalcoholic steatohepatitis (NASH). Estimates in United States are that $17 \%$ to $33 \%$ of all adults have nonalcoholic fatty liver disease, 
whereas $5 \%$ to $17 \%$ have NASH. ${ }^{1-3}$ Although steatosis alone is considered a relatively benign and reversible condition, the development of inflammation represents a key step in liver pathogenesis. Liver inflammation, when untreated, will set the stage for liver fibrosis, cirrhosis, and eventually liver failure. As NASH is mostly asymptomatic, it is necessary to be able to detect hepatic inflammation at an early stage. At present, liver biopsy is the clinical standard for assessing (the progression of) inflammation in fatty livers. However, the procedure has several limitations, including discomfort, longer hospitalization, and risk of serious complications due to its invasiveness. ${ }^{4,5}$ Moreover, diagnosing NASH by biopsy can be associated with sampling errors. In fact, the negative predictive value of a single biopsy for the diagnosis of NASH is estimated at $74 \%{ }^{6}$ Finally, an inadequatelength or fragmented biopsy specimen can make the correct diagnosis even more challenging. ${ }^{7,8}$ Thus, it is extremely important to develop less-invasive techniques for the diagnosis of hepatic inflammation and to discriminate simple steatosis from $\mathrm{NASH}^{7,9}$

Several imaging modalities have been advocated as less-invasive or noninvasive diagnostic tests for simple steatosis, including ultrasonic (US) imaging, computed tomography, magnetic resonance imaging, and recently, also magnetic resonance spectroscopy. ${ }^{9-12}$ Unfortunately, so far these existing imaging techniques have insufficient sensitivity and specificity for distinguishing NASH from noninflammatory fatty liver disease. ${ }^{9}$ Ultrasonic imaging is extensively used clinically because it is safe, versatile, and noninvasive and enables global assessment of organ tissue. ${ }^{9,13}$ Ultrasonic tissue elastography and derivatives are now routinely considered to support diagnosis of (advanced) liver fibrosis but do not provide an independent assessment of the inflammation grade present in the liver. ${ }^{13}$

In US contrast imaging, a contrast agent (typically microbubbles) $)^{14,15}$ is infused into the circulation to assess tissue perfusion. Previously, we have shown that macrophages can be made echogenic by perfluorohexane $(\mathrm{PFH})$ loading while retaining their function as biological sensors for inflammation. ${ }^{16,17}$ Perfluorohexane is a nontoxic, biodegradable, and fluid (ie, nongaseous) US contrast agent that is readily ingested by monocytes/ macrophages on incubation. ${ }^{16,18}$ We found that PFHloaded macrophages were detectable in vivo on US imaging in arterial blood after intravenous injection (ie, they circulate) and specifically interact (roll and attach) with activated endothelium in mouse carotid arteries. ${ }^{17}$
On the basis of these functional findings and in view of the nontoxic nature of $\mathrm{PFH}$, we chose to investigate the utility of PFH-loaded cells for detection of liver inflammation.

In the present study, we hypothesized that the presence and degree of liver inflammation would be reflected in the number and activity of proinflammatory adhesion molecules in the liver vasculature/endothelium. In such a case, a passing bolus of PFH-loaded monocytes will have a higher adhesion affinity, which may delay its wash-out as recorded by US imaging (Figure 1).

Our methodological approach was to inject an intravenous bolus of contrast cells during a continuous liver echo recording and to quantify the wash-out time constant as a measure of the inflammation degree (Figure 1). To evaluate our US PFH-loaded monocyte imaging approach, we performed 2 experiments. In the first, we injected $\mathrm{C} 57 \mathrm{Bl} / 6$ mice intraperitoneally with tumor necrosis factor $\alpha$ (TNF- $\alpha$ ) to simulate vascular endothelial activation. ${ }^{19}$ In the second experiment, $L d r^{-/-}$mice were fed a high-fat/high-cholesterol diet for 1 week to model NASH development. ${ }^{20}$ In both experiments we included control groups and obtained US PFH-loaded monocyte imaging recordings. In this analysis, we focused on the proof of principle for the US PFHloaded monocyte imaging method and included adjunct immunohistochemical findings to discuss its (potential) validity and applicability.

\section{Materials and Methods}

\section{Experimental Animal Models}

All experiments were performed according to Dutch regulations and approved by the Ethical Committee for Animal Welfare of Maastricht University. Mice (20$30 \mathrm{~g}$ ) were housed under standard conditions and given free access to food and water. For the first experiment, TNF- $\alpha(15 \mu \mathrm{g} / \mathrm{kg})$ or phosphate-buffered saline (PBS; control) was injected intraperitoneally in 10- and 12week-old male C57BL/ 6 mice 3 hours before US PFHloaded monocyte imaging measurements (TNF- $\alpha$, $\mathrm{n}=11$; PBS, $\mathrm{n}=11$ ), based on the findings of FoxRobichaud and Kubes. ${ }^{19}$ For the second experiment, 10week-old $\mathrm{Ldlr}^{-/-}$mice (C57BL/6 background strain) were given either chow or a high-fat/high-cholesterol diet for 1 week to induce hepatic tissue inflammation (chow, $\mathrm{n}=10$; high-fat/high-cholesterol, $\mathrm{n}=10$ ). The 
high-fat/high-cholesterol diet consisted of the chow diet supplemented with $20 \%$ milk butter and $1 \%$ cholesterol.

\section{Preparation of the PFH Emulsion}

For preparation of the PFH emulsion, 68\% lecithin (Avanti Polar Lipids, Alabaster, AL) and 2\% 1,2-bis(diphenylphosphino)ethane (Avanti Polar Lipids) were added to 30\% cholesterol (Sigma Aldrich, Steinheim, Germany) and mixed well. ${ }^{16}$ Then evaporation at $60^{\circ} \mathrm{C}$ under nitrogen was performed to prepare a dry lipid mixture, which was followed by the addition of $856 \mu \mathrm{L}$ of Milli-Q (Millipore, Billerica, MA). This mixture was hydrated for 1 hour and vortexed regularly to dissolve the surfactant. Once the sample was fully hydrated, $144 \mu \mathrm{L}$ of $\mathrm{PFH}$ (40\%, C6F14; Sigma Aldrich) was added to the lipid surfactant sample. The PFHsurfactant mixture was emulsified by loading it into a gas-tight syringe fitted to one end of a mini-extruder (Avanti Polar Lipids). By gently pushing the plunger of the filled syringe, the lipid solution was completely transferred into the opposite syringe. This step was reversed and repeated at least 25 times to have a homogeneous lipid solution. ${ }^{16}$ The emulsified mixture was centrifuged for 30 minutes at $2000 \mathrm{~g}$, and the PFH emulsion (pellet) was then dissolved in $2 \mathrm{~mL}$ of PBS, ready to be used for loading bone marrow-derived macrophages. As previously established and described, $\mathrm{PFH}$ is and remains a fluid under the in vitro and in vivo conditions we considered (http://www.rsc.org/learn-chemistry/wiki/Substance:Perfluorohexane). Its boiling temperature is

Figure 1. Background, approach, and hypothesis of US PFH-loaded monocyte imaging for assessment of liver inflammation. Top left, When, during liver (parenchymal) inflammation, the endothelial cells increasingly express adhesion molecules, monocytes/leukocytes will tend to roll and adhere more on the endothelial surface, facing the shearing forces of the bloodstream that naturally wash out these cells from the liver vasculature. Right, As scavengers, monocytes naturally ingest foreign material, such as PFH emulsion droplets, which after incubation will yield monocytes containing conglomerates of PFH droplets, effectively rendering the cells echogenic. On intravenous bolus injection, the echogenic cells will be diluted in the native blood and transported by the bloodstream toward the liver, where the tissue will be transiently highlighted by the echocontrasted cells. Bottom left, With the assumed increased adhesion of the PFH-loaded monocytes with liver inflammation, the time-intensity curves of the US recording are hypothesized to show a reduced wash-out rate: ie, a reduced wash-out time constant.
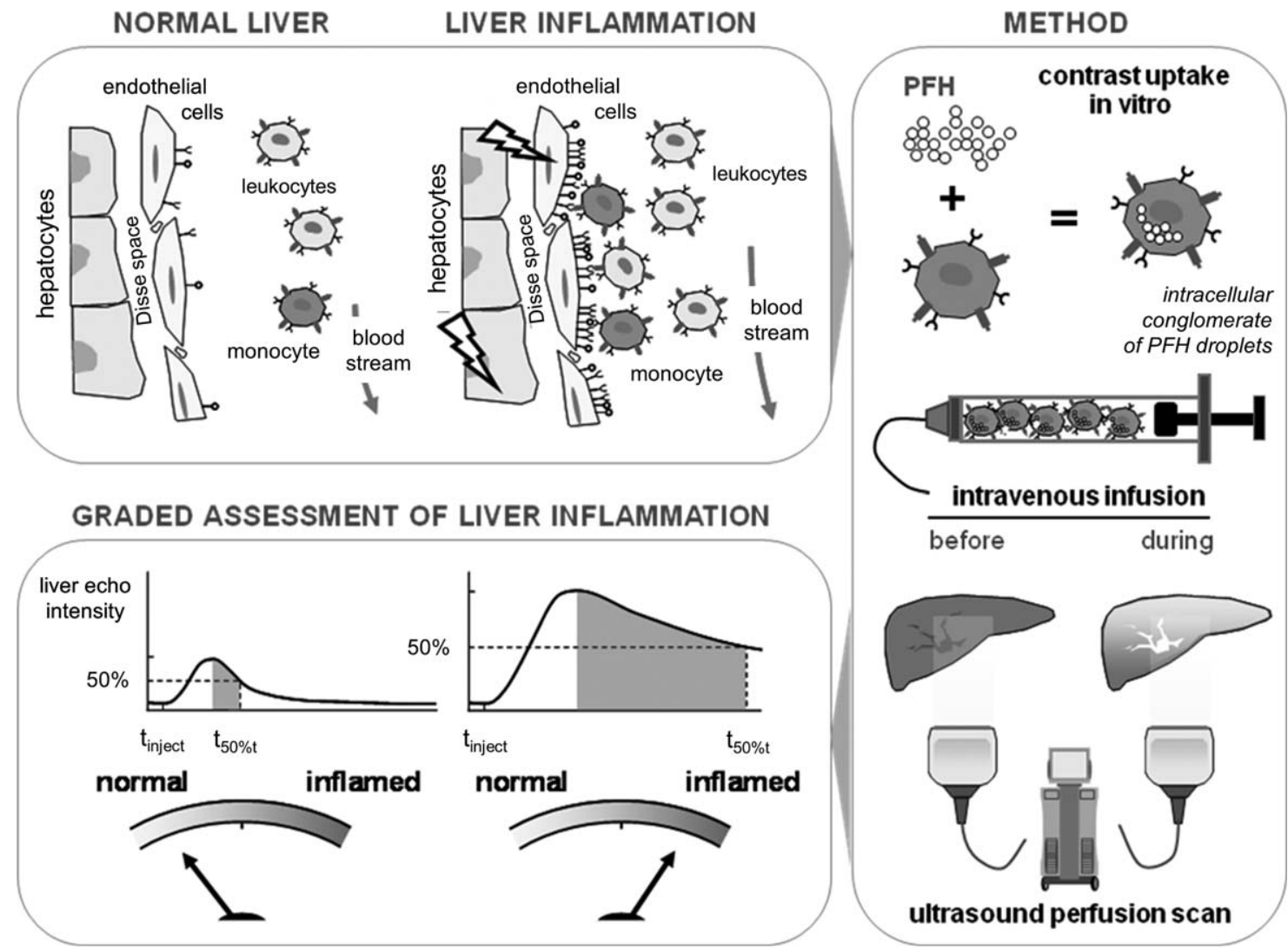
$56^{\circ} \mathrm{C}$, and the mechanical index of the US B-mode imaging we used was far below levels that could lead to evaporation or cavitation. ${ }^{16,17}$ The absence of evaporation/ cavitation is corroborated by our experience, in that in our hands, we have not been able to destroy PFH droplets (monitored by a Vevo 2100 B-mode US system; FUJIFILM VisualSonics, Toronto, Ontario, Canada) deposited on agar (submerged in PBS) ${ }^{16}$ using the Vevo SoniGene sonoporation tool at maximum power delivery $\left(2 \mathrm{~W} / \mathrm{cm}^{2}\right.$ continuous for 30 seconds; our unpublished data). The PFH echogenicity stems from the large difference between its acoustic impedance $(0.875$ MRayl $)$ and that of blood and cells, for example $(\approx 1.5$ MRayl). ${ }^{17}$

\section{Bone Marrow-Derived Macrophages}

Bone marrow-derived macrophages were isolated from the tibias and femurs of C57BL/6 donor mice. Cells were cultured in RPMI-1640 (GIBCO Invitrogen, Breda, the Netherlands) with 10\% heat-inactivated fetal calf serum (Bodinco BV, Alkmaar, the Netherlands), penicillin $(100 \mathrm{U} / \mathrm{mL})$, streptomycin $(100 \mu \mathrm{g} / \mathrm{mL})$, and L-glutamine ( $2 \mathrm{mM}$; all from GIBCO Invitrogen) supplemented with $20 \%$ L929-conditioned medium for 8 to 9 days to generate bone marrow-derived macrophages. After attachment, the macrophages were seeded at $2 \times 10^{6}$ cells per well in 6-well plates for incubation for 16 hours with $4 \% \mathrm{PFH}$ emulsion. ${ }^{16}$ Loaded cells were visually inspected to verify uptake and the integrity of the PFH emulsions in the cells we established previously. ${ }^{16,17}$ Then cells were washed and collected for further use. The PFH-loaded cells effectively act as containers of conglomerates (in vitro cell size $>10 \mu \mathrm{m})^{17}$ of the approximately 300-nm PFH emulsions, ${ }^{21}$ dosedependently enhancing the echogenicity of the loaded cells up to $18 \mathrm{~dB}$, as illustrated previously by light microscopy, flow cytometry, and in vitro US imaging (using a 7.5-MHz linear array). ${ }^{16}$ The properties and echogenic potential of the PFH-loaded cells given as an intravenous bolus have been detailed previously. ${ }^{17}$

\section{Ultrasonic Recording Setup}

We used the Vevo 2100 high-resolution US scanner with an MS-400 linear array transducer (bandwidth, 18$38 \mathrm{MHz}$; operated at $30 \mathrm{MHz}$ ) to record B-mode cine loops. Images were acquired in a single focus zone, with the focus depth in the middle of the liver section considered. Depth-dependent gain was manually adjusted to avoid saturation, which primarily affected the high intensity of the skin layers and border of the liver cross section. This setting remained fixed between experiments while the imaging depth was kept constant. We acquired images triggered by an electrocardiographic (ECG) $\mathrm{R}$ wave detected halfway through the expiration period, minimizing movement artifacts while ensuring an adequate frame rate of 1 per respiratory cycle (Vevo 2100, Physio settings, respiratory gating and ECG gating; minimal respiratory window width, positioned at the middle, flattest portion of the respiratory signal; ECG gate T1 on and set to 0 milliseconds). Consequently, the time for the time-intensity curves was expressed in trigger cycles (each corresponding to a respiratory cycle), and the wash-out time constant as 1 per cycle (further details below).

\section{Experimental Protocol}

Mice were anesthetized 30 minutes after premedication with buprenorphine $(0.03 \mathrm{mg} / \mathrm{kg})$ by inhalation of $3.0 \%$ to $3.5 \%$ isoflurane through a Univentor 410 vaporizer (UNO, Zevenaar, the Netherlands). Subsequently, the chest and belly were shaved and dehaired (VEET; Reckit Benckiser, Hull, England) with the animal's nose and mouth positioned in a breathing mask, maintaining anesthesia by inhaled isoflurane at $1.8 \%$ to $2.5 \%$. Then the right jugular vein was exposed to introduce $(\sim 1 \mathrm{~cm}$ toward the right atrium) a venous cannula (P10, Portex; Smiths Medical International, Kent, England) with an approximately $20-\mathrm{cm}$ external length terminated by a $1-$ $\mathrm{mL}$ syringe with $0.9 \%$ saline via a truncated 30 -gauge needle for flushing. Subsequently, the anesthetized and cannulated mouse was transferred to the Vevo 2100 scanning platform. The paws were carefully positioned and fixated on electrodes for ECG and plethysmographic registration, ensuring good contact by application of electrode gel. The rectal temperature was monitored and manually regulated within $36.8^{\circ} \mathrm{C}$ to $37.2^{\circ} \mathrm{C}$ by an infrared lamp.

After positioning of the animal on the scanning platform, the US transducer was placed just above the animal, with a 2- to 5-mm-thick US gel mass in between (without air bubbles). Positioning was transverse and initially roughly between lower ribs and bowels. The $x-y$ manipulator of the US setup was then used to adjust for the maximum observed liver area with no or minimal clutter from surrounding structures. Subsequently, during a 2- to 5-minute period for stabilization of the respiratory rate under inhaled isoflurane ( $40-60$ cycles $/ \mathrm{min}$ ), 
the injectate syringe was prepared: ie, re-emulsification of the cell emulsion in an Eppendorf container kept at $37^{\circ} \mathrm{C}$ and uptake of $2-3 \times 10^{6}$ cells in $200 \mu \mathrm{L}$ into a 1$\mathrm{mL}$ syringe with a truncated needle to fit the jugular vein cannula. During and directly after connecting the syringe to the cannula, settling of the cell emulsion was prevented by rocking the syringe until injection commenced. The echo recording was started by freezing and unfreezing the acquisition. Twenty trigger cycles (ie, respiratory periods) were counted off to establish a baseline, after which the injectate was injected smoothly over 50 to 60 trigger cycles ( $\approx 1$ minute). The recording was stopped after 400 trigger cycles and saved to the hard disk on the Vevo 2100 system. Recordings were exported as avi files to an external hard disk for offline processing.

After the US measurements (as described above), the animals were euthanized by cervical dislocation. Collection of blood and tissue specimens, biochemical determination of lipids in the liver, liver histologic analysis, RNA isolation, complementary DNA synthesis, and quantitative polymerase chain reaction were performed as described previously. ${ }^{22}$

\section{Ultrasonic Video Processing}

The US video recordings were processed by a proprietary algorithm written by written by APH (MATLAB R2013b, The Mathworks, Natick, MA), according to the following steps (in the order of execution): (1) Intensity calibration was read from the calibration bar in the first B-mode frame and applied in reverse to obtain linearly scaled intensity values (ie, to decompress the normally compressed grayscale range, which favors visual evaluation but is unsuited to quantifying changes in intensity). (2) Based on the first B-mode frame, a region of interest (ROI) was selected to maximize the area of the liver section considered while avoiding the borders of the liver parenchyma. (3) Subsequently, spatial and temporal smoothing was performed by a 3-dimensional uniform running window with dimensions of $(0.1 \times \mathrm{ROI}$ width $) \times(0.1 \times$ ROI depth $) \times 9$ sample points (ie, 9 respiratory cycles) in width, depth, and time, respectively. (4) Based on the smoothed data, for a $3 \times 3$ grid, time-intensity curves and their average were extracted for an intermediate evaluation (see below). (5) A time base marker was then manually set to define the baseline (ie, preinjection) value. The baseline value was subtracted from the time-intensity curve to isolate the transient response from the steady-state background intensity. The resultant time-intensity curves and processing parameters were saved to a hard disk.

At this point, we visually evaluated the US $\mathrm{PFH}$ loaded monocyte imaging recordings and excluded recordings with no identifiable peak in the average timeintensity curve $(n=14)$ or with premature ending due to hemodynamic instability of the mouse $(\mathrm{n}=5)$. Identification of the time-intensity curve peak is a necessary requirement for determining the wash-out time constant (next paragraph). The time-intensity curve peak was assumed to occur within 100 trigger cycles from the start of injection (illustrated in Figure 2, B and C). All mice showed some cardiopulmonary response during of immediately on injection: specifically, a transient decrease in the respiratory rate. In 2 particular cases, this response led to a disruption of the triggering, signal and the recording stopped automatically (ie, by scanner default). In those cases, another recording commenced immediately and provided a sufficient wash-out period to perform the analysis on. Final numbers of mice per group included in this analysis of US PFH-loaded monocyte imaging were 8 for TNF- $\alpha$ and 7 for PBS controls and 5 for the high-fat/high-cholesterol diet and 6 for chow controls.

For characterization of the wash-out, we assumed the imaged liver blood pool to behave as a single compartment with a first-order wash-out response in time, starting from the peak echo intensity level. Considering that (1) our PFH-loaded cells are relatively inert (no notable disintegration in vitro after 24 hours ${ }^{16}$; (2) substantial clearance/transgression of the cells out of the circulation is unlikely to occur within the time frame observed ( $\approx 5$ minutes); and ( 3 ) the circulation is a single compartment in which the bolus will dilute depending on circulatory flow, ${ }^{23}$ this first-order model appears valid. Accordingly, we quantified the wash-out by a simple exponential decay time constant. To obtain an estimate of the wash-out decay time constant that was representative of the liver section imaged, the following steps were taken: (1) The down slope of the average time-intensity curve was manually defined by drag-anddrop markers starting at the peak intensity and ending close to 0 . If after the first decay, a rise in intensity was observed, the start of the rise was taken as the end of the decay (Figure 2, B and C, block arrow 2). (2) Assuming the first-order decay model, the decay characteristics (amplitude and wash-out time constant) were obtained 
for the average time-intensity curve (online supplemental Figure S1, left panel). (3) For a grid, with an interspacing of one-fourth of the corresponding smoothing span in width and depth, time-intensity curves were evaluated (eg, for a smoothing span $20 \times 12$ wide $\times$ deep, $5 \times 3$ time-intensity curves were obtained). Only timeintensity curves with an identifiable peak (same requirement as described above) and a wash-out time constant within the range 0.5 to 2 of that of the average timeintensity curve were accepted (online supplemental Figure S1, middle panel). (4) Finally, the histogram and median of the wash-out time constants were displayed, and the results were saved to the hard disk. The median wash-out time constant was taken as representative of the US PFH-loaded monocyte imaging measurement considered (online supplemental Figure S1, right panel).

In a pilot data set, we evaluated the variability due to ROI selection to assess the impact of artifactual motion and consequent intensity changes due to edge effects. We found a variability in the time constant of about $30 \%$, which compared favorably with the rate of greater than $50 \%$ reported by Ignee et $\mathrm{al}^{24}$ although those investigators did not evaluate the wash-out time constant as such. We have not conducted a laboratory bench validation against a reference standard method for the wash-out time constant. Consequently, the validity of the time constant estimates necessarily relied on the statistical power of multiple realizations over the ROI and taking the median value.

\section{Statistical Analysis}

Data were analyzed with Prism version 4.0.3 software (GraphPad Software, La Jolla, CA). Groups were compared by an unpaired $t$ test for comparing 2 groups. The data are presented as mean \pm standard error of the mean, unless noted otherwise. Differences between experimental and respective control groups were considered significant at $P<.05, P<.01$, or $P<.001$.

Figure 2. A, In TNF- $\alpha$-injected mice ( $n=8$ ), the wash-out time constant (1 per cycle) was clearly decreased compared with PBS controls ( $n=7$ ): ie, the TNF- $\alpha$ group showed slower wash-out ( $\left.{ }^{* \star} P=.0006\right)$. Box plots show the median, interquartile range, and minimum-maximum range. $\mathbf{B}$ and $\mathbf{C}$, Time-intensity curves and manually defined wash-out ranges for 2 normal and 2 slowed wash-outs, respectively, with the time axis given in (respiratory) cycles. The dots mark the start and end of the selected wash-out data for calculation of the time constants, assuming a first-order decay curve (gray dotted lines). The numbers indicate the corresponding time constants (in 1/cycles). Block arrow 1 illustrates a nonmonotonous rise in intensity during injection. Block arrow 2 illustrates selection of the end value of the decay in case a secondary rise in intensity follows.

A

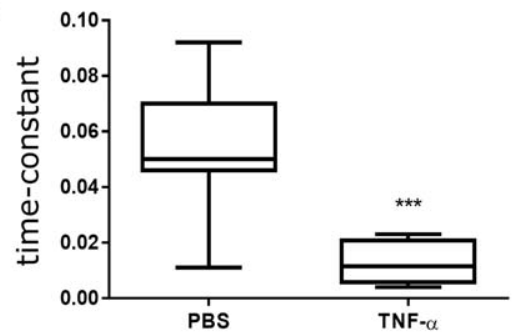

B

normal wash-out
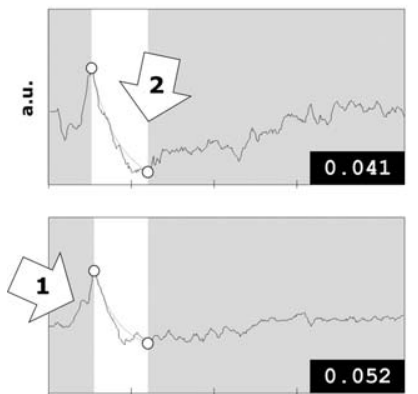

C delayed wash-out
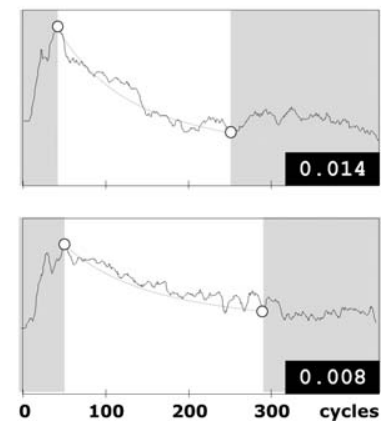


\section{Results}

\section{Ultrasonic PMI Results 3 Hours After}

\section{Intraperitoneal TNF- $\alpha$ Injection}

To test whether US PFH-loaded monocyte imaging could be used to detect inflammation in the liver on direct endothelial stimulation, $\mathrm{C} 57 \mathrm{Bl} / 6$ mice were injected intraperitoneally with TNF- $\alpha$ or PBS and assessed by US PFH-loaded monocyte imaging 3 hours later. As indicated in Figure 2A, wash-out was significantly slower in the TNF- $\alpha$ mice with a directly activated endothelium compared to controls; wash-out constants were $0.013 \pm 0.003 \quad(n=8)$ and $0.054 \pm 0.009(n=7)$, respectively $(P=.0006)$. Figure $2, \mathrm{~B}$ and $\mathrm{C}$, illustrates time-intensity curves and manually defined wash-out ranges for 2 normal and 2 slowed wash-outs, respectively.
Tumor Necrosis Factor $\alpha$ Injection Does Not Induce a Hepatic Inflammatory Response

Mice were euthanized 3 hours after intraperitoneal injection of TNF- $\alpha$, and livers were harvested for investigation of the development of hepatic inflammation. To do so, liver sections were stained with antibodies against infiltrating macrophages (Mac-1) and neutrophils (antineutrophil antibody [NIMP]). Although a trend was found toward increased infiltrating inflammatory cells after TNF- $\alpha$ injection, no significant difference was observed compared to control mice $(n=10$ for each group; Figure 3, A and B). In addition, no difference in hepatic gene expression of $T n f-\alpha$, integrin $\alpha \mathrm{M}$ (Itgam), intracellular adhesion molecule (Icam) and vascular cell adhesion molecule (Vcam) was found between TNF- $\alpha$ injected and control mice $(n=10$ for each group; Figure $3 \mathrm{C}$ ). These data indicate that 3 to 4 hours after

Figure 3. Parameters of hepatic inflammation in PBS- and TNF- $\alpha$-injected mice. A, Liver sections were stained for infiltrating macrophages (Mac1) and neutrophils (NIMP), and the positive cells were counted. B. Representative images of Mac-1 staining (original magnification $\times 200$ ) in PBS- and TNF- $\alpha$-injected mice. C, Gene expression analysis of Tnf- $\alpha$, Itgam, Icam, and Vcam. Gene expression data were set relative to PBSinjected mice. Bar graph data are given as mean and SEM; for each group, $n=10$.
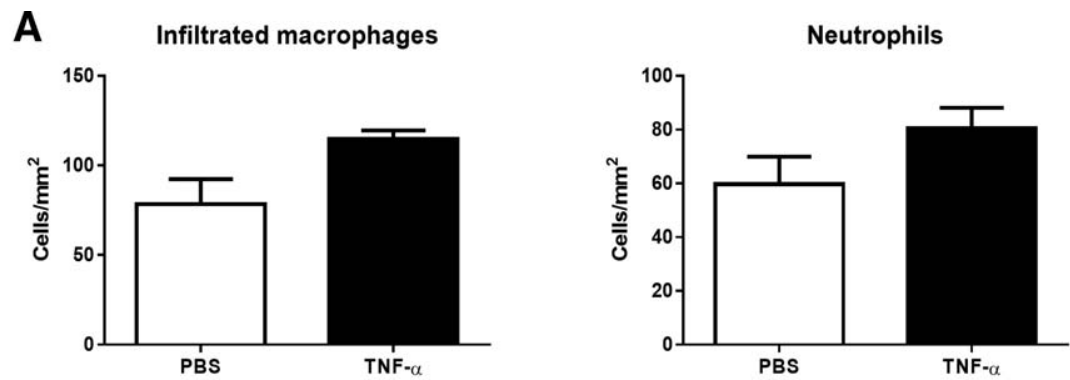

B

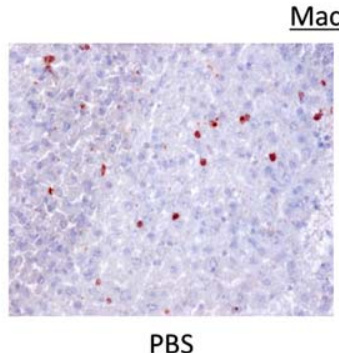

Mac-1 staining
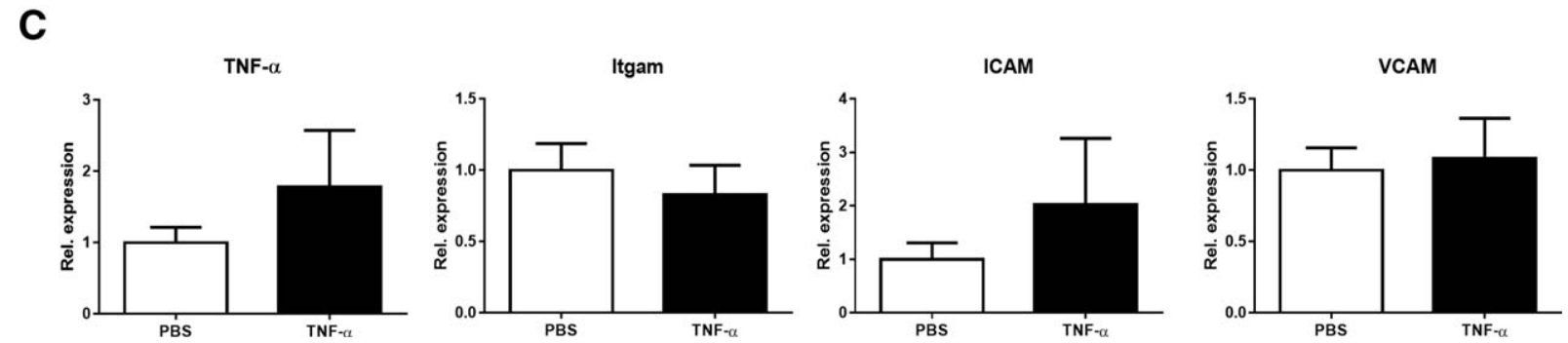
intraperitoneal injection of TNF- $\alpha$, the hepatic tissue inflammatory response was (still) absent.

\section{Ldlr $^{-/-}$Mice Develop Hepatic Steatosis and} Inflammation After 1 Week of a High-Fat/HighCholesterol Diet

After 1 week of the high-fat/high-cholesterol diet, $\mathrm{Ldlr}^{-/-}$mice developed hepatic steatosis, as indicated by the increased hepatic levels of triglycerides, cholesterol, and free fatty acids compared to $\mathrm{Ldlr}^{-/-}$mice fed the chow diet $(n=10$ for each group; online supplemental Figure S2A). In addition, CD68 staining (for Kupffer cells) was performed, revealing a clear difference between $\mathrm{Ldlr}^{-1-}$ mice fed the high-fat/high-cholesterol diet and mice fed chow. As expected, in the $\mathrm{Ldlr}^{-/-}$ mice fed the high-fat/high-cholesterol diet, the Kupffer cells were more swollen and foamy compared to Kupffer cells in the chow-fed animals (online supplemental Figure S2B).

To investigate the development of hepatic inflammation, liver sections were stained with antibodies against several inflammatory markers, including macrophages (Mac-1) and neutrophils (NIMP). Higher numbers of infiltrating macrophages and neutrophils were observed in the livers of the $\mathrm{Ldlr}^{-1-}$ mice fed the highfat/high-cholesterol diet compared to the $\mathrm{Ldlr}^{-/-}$mice fed the chow diet ( $n=10$ for each group; Figure 4A), as further illustrated by representative images from the Mac-1 staining for infiltrating macrophages and neutrophils (Figure 4B). These findings were confirmed by

Figure 4. Parameters of hepatic inflammation in chow- and high-fat/high-cholesterol (HFC) diet-fed mice. A, Liver sections were stained for infiltrating macrophages (Mac-1) and neutrophils (NIMP), and the positive cells were counted. B, Representative images of Mac-1 staining (original magnification $\times 200$ ) after 1 week of the chow or high-fat/high-cholesterol diet in Ldlr ${ }^{-1-}$ mice. C. Gene expression analysis of Tnf- $\alpha$, Itgam, Icam, and Vcam. Gene expression data were set relative to chow-fed mice. Bar graph data are given as mean and SEM. ${ }^{\star \star} P<.01$; ${ }^{\star \star \star} P<.001$; for each group, $\mathrm{n}=10$.

A

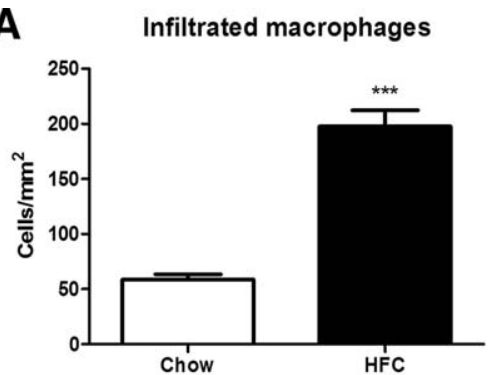

Neutrophils

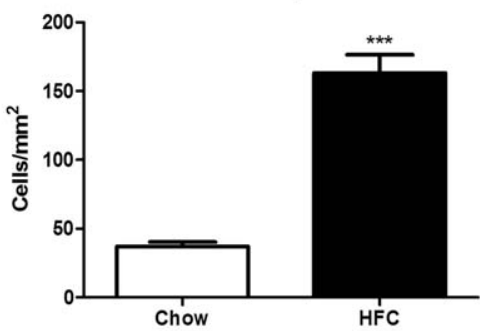

B

Mac-1 staining

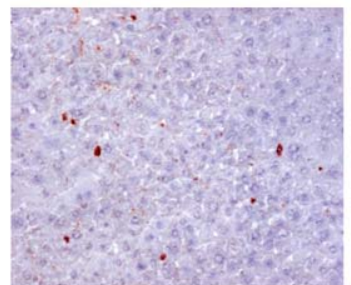

Chow

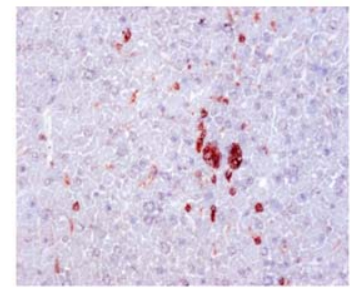

HFC

\section{C}
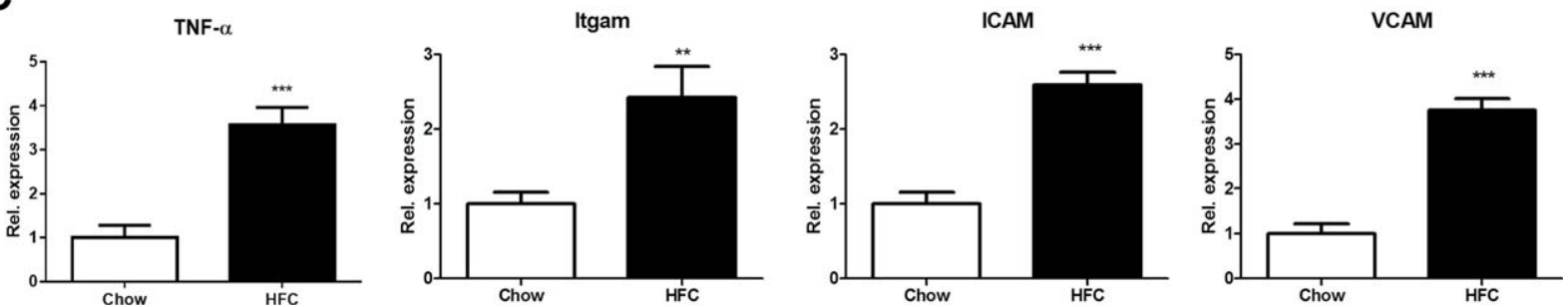
increased hepatic gene expression of Tnf- $\alpha$, Itgam, Icam, and $V c a m$ in the $\mathrm{Ldlr}^{-/-}$mice fed the high-fat/highcholesterol diet compared to the chow-fed $\mathrm{Ldlr}^{-/-}$ mice (Figure 4C). Taken together, these data indicate that a 1-week high-fat/high-cholesterol diet results in (detectable) liver tissue (ie, parenchymal) inflammation.

\section{No Difference in Wash-out of PFH-Loaded Monocytes in Ldlr ${ }^{-/-}$Mice After 1 Week of a High-Fat/High-Cholesterol Diet Compared to Controls}

Figure 5 shows that there was no significant difference in wash-out time constants between the mice fed the high-fat/high-cholesterol diet and the control mice fed the chow diet. Wash-out time constants were $0.050 \pm$ $0.017(\mathrm{n}=5)$ and $0.048 \pm 0.006(\mathrm{n}=6)$, respectively $(P=.91)$.

\section{Discussion}

This study demonstrates proof of the principle for the use of perfluorohexane-loaded monocytes as a contrast agent and US B-mode cine recording as an imaging tool for selective detection of liver inflammation. Direct endothelial stimulation led to the hypothesized decrease in the contrast wash-out time constant: ie, a slower wash-out. Direct endothelial stimulation did not lead to increased (parenchymal) inflammatory tissue markers. In $\mathrm{Ldlr}^{-/-}$mice fed the 1-week high-fat/high-cholesterol diet (developing NASH), we did not detect a slower wash-out, although immunohistologic markers showed a clear inflammatory tissue phenotype. Below, we will critically discuss our findings.

Figure 5. No difference in the US PFH wash-out time constant between mice after 1 week of the high-fat/high-cholesterol (HFC) diet $(n=5)$ and chow diet $(n=6) ; P=.91$. Box plots show the median, interquartile range, and minimum-maximum range.

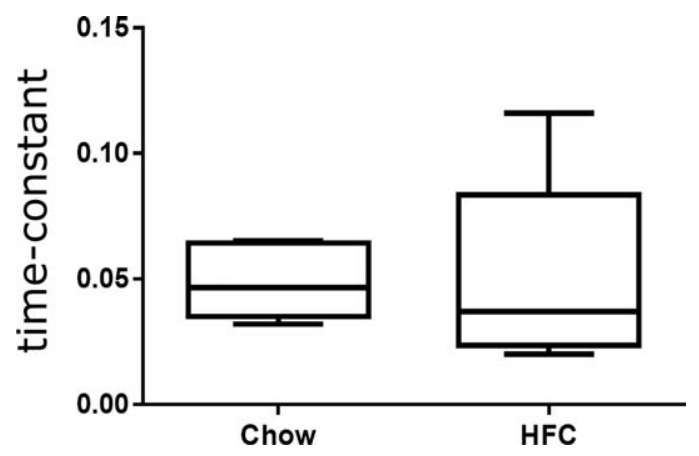

Our findings in the TNF- $\alpha$-stimulated mice are in line with the increased expression of vascular adhesion molecules in the liver as reported by Fox-Robichaud and Kubes ${ }^{19}$ and the increased adherence of native leukocytes at the carotid artery endothelium as we recently reported. ${ }^{17}$ The increased tendency of the PFH-loaded macrophages to adhere leads to a prolonged residence time (before a cell detaches again and is carried further by the bloodstream) in the liver vasculature ${ }^{25}$ and hence could well explain the hypothesized decreased wash-out decay time constant we observed in the TNF- $\alpha$ stimulated mice.

Iijima et $\mathrm{al}^{15}$ obtained US time-intensity curves in patients with NASH and simple steatosis, using a microbubble contrast agent with no (inflammation-) specific binding affinity with a focus on delayed enhancement of liver tissue. ${ }^{15}$ Those investigators reported evidence of a faster wash-out in a patient with NASH compared to a patient with simple steatosis (cf Iijima et al, ${ }^{15}$ Figure 3). Unfortunately, their analyses further focused on the intensity level rather than wash-out timing and lacked data on the correlation with the degree of inflammation (also see Cobbold et al, "Discussion"). Moreover, their use of a nonspecific contrast agent is at odds with the pathophysiologic basis of our approach (Figure 1).,19

In agreement with our previous observations, ${ }^{20}$ $\mathrm{Ldlr}^{-1-}$ mice develop steatosis and hepatic tissue inflammation on short-term (ie, 1-week) high-fat/highcholesterol feeding, as reflected by our liver lipid analysis and immunohistologic findings. In addition, the swollen appearance of Kupffer cells with the high-fat/high-cholesterol diet resembles foamy macrophages, as observed during atherosclerosis, indicating the accumulation of intracellular lipids in activated Kupffer cells, leading to inflammatory signals. However, to what extent this short-term high-fat/high-cholesterol diet leads to (detectable) endothelial expression of adhesion molecules is not yet known. In this setting for in vivo determination of hepatic inflammation, we did not find a decreased wash-out time constant in high-fat/high-cholesterol-fed mice compared to controls.

One explanation for this negative US PFH-loaded monocyte imaging result in the high-fat/high-cholesterol-fed mice pertains to the limited specificity of our immunohistologic analysis. We determined gene expression profiles in homogenates of liver tissue excised and stored after the US PFH-loaded monocyte imaging recordings. Given the estimation that 
$70 \%$ to $85 \%$ of the liver volume is taken up by hepatocytes, ${ }^{26}$ one may expect that the parenchymal contribution to the expression of cell surface adhesion molecules assessed in the homogenates may dominate over that of the vascular endothelium. Consequently, this factor likely limited us in assessing modulation of endothelial adhesion molecule expression in the mice fed the high-fat/high-cholesterol diet.

Another, more fundamental, issue may be that in our high-fat/high-cholesterol-fed mice, the level of tissue/parenchymal inflammation did not (yet) cause sufficient endothelial adhesion molecule expression, hence staying below the detection limit of our US PFHloaded monocyte imaging method. This factor does prompt the question of what level of parenchymal inflammation sets off increased expression of adhesion molecules at the endothelial surface in the liver vasculature (ie, around the Disse space). From atherosclerosis research, it is known that inflammatory cells inside the vessel wall release cytokines that trigger endothelial expression of adhesion molecules, such as the ICAM and VCAM integrins. ${ }^{27}$ In the liver, a similar chain of inflammatory events is believed to occur, ${ }^{19,28}$ but differences in endothelial adhesion molecule expression profiles between locations (presinusoidal, sinusoidal, and venular) are relevant to consider. ${ }^{19}$ An additional analysis of the present data showed 2-fold expression of $V c a m$ in the mice fed the high-fat/high-cholesterol diet directly compared to TNF- $\alpha$-stimulated mice $(P=.003$, 2-sample $t$ test). However, leukocyte recruitment in the liver vasculature is not solely governed by VCAM to $\alpha_{4}$-integrin interaction. ${ }^{29}$ A similar but tentative 2-fold increased expression of Tnf- $\alpha$ in our highfat/high-cholesterol-fed mice compared to our TNF$\alpha$-stimulated mice $(P=.096,2$-sample $t$ test $)$ may have been less-directly linked to adhesion, given the dominant parenchymal contribution of this cell surface marker, as already discussed above. There were similar expression levels of Itgam and Icam between the groups, which do not support the interpretation of the negative US PFH-loaded monocyte imaging finding in the high-fat/high-cholesterol experiment. It should also be noted that the (increased) size and deformability of PFH-loaded cells (as described previously ${ }^{16,18}$ may have an effect on wash-out dynamics. We have found no evidence in the literature to substantiate differences in vascular dimensions and perfusion between our mouse models. $^{24}$
Last, the time dependence of the relationship between parenchymal and endothelial inflammation is worth considering, given our findings. As we evaluated US PFH-loaded monocyte imaging in $\mathrm{Ldlr}^{-/}$mice after a 1-week high-fat/high-cholesterol diet (ie, with NASH still developing), it might be that the endothelial inflammation, when assuming it follows parenchymal inflammation, had yet to become detectable. To the best of our knowledge, this area, where the biophysics and kinetics of cell migration and paracrine signaling play major roles, has not been explored to a great extent, at least not in the field of nonalcoholic fatty liver disease. $^{2,19}$ Given the dynamic aspect of signaling and disease development, the timing of US PFH-loaded monocyte imaging (and possible adjunct molecular imaging) needs to be more carefully considered and controlled in future studies.

For the characterization of the wash-out, we assumed that the imaged liver blood pool was a single compartment with a first-order wash-out response in time, starting from the maximum echo intensity level. In the time-intensity curves illustrated in Figure 2, secondpass effects appeared to occur as a result of recirculation, which was obviously present. However, considering the potential factors influencing the time-intensity curve away from the initial wash-out period, we did not, at this point, consider using a more comprehensive perfusioncirculation model suitable. Therefore, in our timeintensity curve analysis, we explicitly assumed the initial wash-out period to be a simple exponential decay. Our time-intensity curves, as exemplified in Figure 2, suggest that this approach is reasonable and practicable.

We obtained liver echo intensity frames triggered on the respiratory plethysmogram. This method is a necessary and effective way to reduce motion/intensity artifacts due to thorax and diaphragm movement. ${ }^{30}$ The sampling rate appeared sufficient, whereas decay curves spanned at least 40 trigger cycles (Figure 2, B and C). Within each respiratory cycle, we obtained one frame based on the ECG $\mathrm{R}$ wave and the gate set to the minimal duration. However, the Vevo 2100 trigger unit uses an ECG gate width as a fraction of the respiratory period. Consequently, for animals with a relatively long respiratory period with respect to the R-R interval, this factor occasionally led to acquisition of 2 frames per respiration period. We could not easily correct for this error because we did not have the ECG and respiratory signal available in the avi format videos we analyzed. Because 
these errors were infrequent and did not consistently occur, we consider their impact on the time constant estimates limited.

We used manual injections to perform the US PFH-loaded monocyte imaging measurements. An infusion pump would clearly produce more even inflow over the 50- to 60-cycle injection period. However, settling of the cell emulsion was an issue: not rocking the syringe led to fatal embolization of the lungs in preliminary experiments using jugular vein access. ${ }^{17}$ Unsteady injection might have led to nonmonotonous rises in liver intensity (illustrated by block arrow 1 in Figure 2, B and C). Nevertheless, our manual injections of the 2 to $3 \times 10^{6}$ cells in $200 \mu \mathrm{L}$ did lead to clearly identifiable peak intensities in most mice, to serve as valid (amplitude and time) references for the wash-out phase of interest; the time-intensity curve course preceding the identified peak is irrelevant for our approach (illustrated by block arrow 2 in Figure 2, B and C).

We could not identify a peak intensity in 14 mice, which deserves some consideration. It might be that in these cases, liver perfusion was insufficient to lead to a detectable time-intensity curve. Alternatively, loss of PFH-loaded cells to adhesion outside the liver may explain insufficient contrast enhancement in these animals. However, we did not find a notable difference in the number of time-intensity curves with no identifiable peak between the TNF- $\alpha$ and high-fat/high-cholesterol experiments ( $\mathrm{n}=6$ and 8 , respectively), although one may expect a steal effect to be greater in TNF- $\alpha$-treated mice. The corresponding adhesion molecule expression profiles of the mice with no identifiable peak were around the average value of the respective whole group ( $n=10$ in each of the groups). For the immunohistologic analyses, therefore, it is justified that we did use all data available.

Although we optimized the injectate volume and concentration in initial experiments, we still lost 5 US PFH-loaded monocyte imaging outcomes because of hemodynamic instability (see "Materials and Methods"). As the dose was such that no obvious intensity increase was noticeable by eye on the scanner (however measurable), it might have been that conglomerates of cells did reach the lungs and caused embolization there or farther downstream.

In this study, we did not assess hemodynamic differences between experimental groups (eg, by contrast perfusion scanning with nonspecific affinity to inflammatory adhesion molecules). Therefore, we cannot exclude or discriminate a hemodynamic influence on the observed wash-out time constants but only assume that in our models with acute endothelial stimulation with TNF- $\alpha$ and developing NASH $\left(\mathrm{Ldll}^{-/-}\right.$ mice fed the 1-week high-fat/high-cholesterol diet), these advanced effects, which may concur with advanced fibrosis, are only minor. ${ }^{2}$ On visual inspection, we did not notice a difference between the histogram shapes of TNF- $\alpha$-stimulated and of Ldlr-/- high-fat/highcholesterol-fed mice, which might have indicated a difference in wash-out kinetics.

Another important limitation was that we did not assess in vivo endothelial adhesion molecule expression directly (ie, by targeted microbubbles), which would have enabled us to corroborate our positive US PFHloaded monocyte imaging findings in the TNF- $\alpha$ stimulated mice. We specifically chose not to avoid possible interference between US PFH-loaded monocyte imaging and targeted microbubble measurements because a preceding measurement may influence adhesion molecule availability or affinity for the subsequent measurement. In future experiments, adhesion molecule expression on the endothelium could be assessed by immunofluorescence imaging. With the use of 2-photon laser-scanning microscopy, it should be (after staining and fixation) possible to identify the endothelial/vascular aspect in 3 dimensions in intact liver tissue and assess the presence and density of adhesion molecules, such as ICAM- 1 and VCAM-1. ${ }^{31}$

Lastly, we did not assess liver tissue damage due to administration of the PFH-loaded macrophage suspension. Dirven et $\mathrm{al}^{14}$ reported liver lesions on microbubble administration, but only after 3 hours. Given that we used $\mathrm{PFH}$, which is fluid under the conditions considered, ${ }^{17}$ and that the US PFH-loaded monocyte imaging procedure duration was limited ( $<15$ minutes), we consider damage due to PFH irrelevant in the present analysis. At this stage, the longer-term effect of administering monocytes/macrophages on the inflammatory process in the liver remains of concern and deserves further study, before sequential US PFH-loaded monocyte imaging measurements can be considered tools for studying progression/regression of NASH (in experimental mice). ${ }^{2}$

As it stands, we have introduced and obtained proof of concept for the much needed minimally invasive and selective detection of inflammation in nonalcoholic fatty 
liver disease by US PFH-loaded monocyte imaging. ${ }^{2,9}$ Ultrasonic PFH-loaded monocyte imaging is able to detect vascularly expressed inflammatory adhesion molecules in the mouse liver on direct endothelial stimulation. However, in our model for early developing $\mathrm{NASH}$, we did not detect inflammation by US PFHloaded monocyte imaging, which (apart from methodological limitations) may suggest that the time-dependent relationship between parenchymal and endothelial inflammation remains a fundamental issue to be addressed.

\section{References}

1. Clark JM, Diehl AM. Defining nonalcoholic fatty liver disease: implications for epidemiologic studies. Gastroenterology 2003; 124: 248-250

2. Cohen JC, Horton JD, Hobbs HH. Human fatty liver disease: old questions and new insights. Science 2011; 332:1519-1523.

3. McCullough AJ. The clinical features, diagnosis and natural history of nonalcoholic fatty liver disease. Clin Liver Dis 2004; 8:521-533.

4. Piccinino F, Sagnelli E, Pasquale G, Giusti G. Complications following percutaneous liver biopsy: a multicentre retrospective study on 68,276 biopsies. J Hepatol 1986; 2:165-173.

5. Terjung B, Lemnitzer I, Dumoulin FL, et al. Bleeding complications after percutaneous liver biopsy: an analysis of risk factors. Digestion 2003; 67:138-145.

6. Barsic N, Lerotic I, Smircic-Duvnjak L, Tomasic V, Duvnjak M. Overview and developments in noninvasive diagnosis of nonalcoholic fatty liver disease. World J Gastroenterol 2012; 18:3945-3954.

7. Al Knawy B, Shiffman M. Percutaneous liver biopsy in clinical practice. Liver Int 2007; 27:1166-1173.

8. Ratziu V, Charlotte F, Heurtier A, et al. Sampling variability of liver biopsy in nonalcoholic fatty liver disease. Gastroenterology 2005; 128: 1898-1906.

9. Cobbold JF, Patel D, Taylor-Robinson SD. Assessment of inflammation and fibrosis in non-alcoholic fatty liver disease by imaging-based techniques. J Gastroenterol Hepatol 2012; 27:1281-1292.

10. Browning JD, Horton JD. Molecular mediators of hepatic steatosis and liver injury. J Clin Invest 2004; 114:147-152.

11. Szczepaniak LS, Babcock EE, Schick F, et al. Measurement of intracellular triglyceride stores by $\mathrm{H}$ spectroscopy: validation in vivo. Am J Physiol 1999; 276:E977-E989.

12. Thomas JA, Aithal GP. Monitoring liver function during methotrexate therapy for psoriasis: are routine biopsies really necessary? Am J Clin Dermatol 2005; 6:357-363.

13. Orlacchio A, Bolacchi F, Antonicoli M, et al. Liver elasticity in NASH patients evaluated with real-time elastography (RTE). Ultrasound Med Biol 2012; 38:537-544.
14. Dirven HA, Rasmussen H, Johnsen H, Videm S, Walday P, Grant D. Intestinal and hepatic lesions in mice, rats, and other laboratory animals after intravenous administration of gas-carrier contrast agents used in ultrasound imaging. Toxicol Appl Pharmacol 2003; 188:165175.

15. Iijima $\mathrm{H}$, Moriyasu F, Tsuchiya $\mathrm{K}$, et al. Decrease in accumulation of ultrasound contrast microbubbles in non-alcoholic steatohepatitis. Hepatol Res 2007; 37:722-730.

16. Kornmann LM, Curfs DM, Hermeling E, et al. Perfluorohexaneloaded macrophages as a novel ultrasound contrast agent: a feasibility study. Mol Imaging Biol 2008; 10:264-270.

17. Kornmann LM, Zernecke A, Curfs DM, et al. Echogenic perfluorohexane-loaded macrophages adhere in vivo to activated vascular endothelium in mice: an explorative study. Cardiovasc Ultrasound 2015; $13: 1$.

18. Flaim SF. Pharmacokinetics and side effects of perfluorocarbon-based blood substitutes. Artif Cells Blood Substit Immobil Biotechnol 1994; 22: 1043-1054.

19. Fox-Robichaud A, Kubes P. Molecular mechanisms of tumor necrosis factor alpha-stimulated leukocyte recruitment into the murine hepatic circulation. Hepatology 2000; 31:1123-1127.

20. Bieghs V, Van Gorp PJ, Wouters K, et al. LDL receptor knock-out mice are a physiological model particularly vulnerable to study the onset of inflammation in non-alcoholic fatty liver disease. PLoS One 2012; 7:e30668.

21. Couture O, Bevan PD, Cherin E, Cheung K, Burns PN, Foster FS. A model for reflectivity enhancement due to surface bound submicrometer particles. Ultrasound Med Biol 2006; 32:1247-1255.

22. Hendrikx T, Jeurissen ML, Bieghs V, et al. Hematopoietic overexpression of Cyp27al reduces hepatic inflammation independently of 27hydroxycholesterol levels in Ldlr(-/-) mice. J Hepatol 2015; 62:430436.

23. Stewart GN. Researches on the circulation time and on the influences which affect it. J Physiol 1897; 22:159-183.

24. Ignee A, Jedrejczyk M, Schuessler G, Jakubowski W, Dietrich CF. Quantitative contrast enhanced ultrasound of the liver for time intensity curves: reliability and potential sources of errors. Eur J Radiol 2010; 73:153-158.

25. Ley K, Laudanna C, Cybulsky MI, Nourshargh S. Getting to the site of inflammation: the leukocyte adhesion cascade updated. Nat Rev Immunol 2007; 7:678-689.

26. Zhou Z, Xu MJ, Gao B. Hepatocytes: a key cell type for innate immunity. Cell Mol Immunol 2016; 13:301-315.

27. Nakashima Y, Raines EW, Plump AS, Breslow JL, Ross R. Upregulation of VCAM-1 and ICAM-1 at atherosclerosis-prone sites on the endothelium in the ApoE-deficient mouse. Arterioscler Thromb Vasc Biol 1998; 18:842-851.

28. Bieghs V, Rensen PC, Hofker MH, Shiri-Sverdlov R. NASH and atherosclerosis are two aspects of a shared disease: central role for macrophages. Atherosclerosis 2012; 220:287-293. 
Reesink et al—Ultrasonic Perfluorohexane-Loaded Monocyte Imaging of Liver Inflammation

29. Lee WY, Kubes P. Leukocyte adhesion in the liver: distinct adhesion paradigm from other organs. J Hepatol 2008; 48:504 512 .

30. Averkiou M, Lampaskis M, Kyriakopoulou K, et al. Quantification of tumor microvascularity with respiratory gated contrast enhanced ultrasound for monitoring therapy. Ultrasound Med Biol 2010; 36:6877.

31. Curaj A, Wu Z, Fokong S, et al. Noninvasive molecular ultrasound monitoring of vessel healing after intravascular surgical procedures in a preclinical setup. Arterioscler Thromb Vasc Biol 2015; 35:1366-1373. 